\title{
EDITORIAL
}

\section{The Cochrane Collaboration's Complementary Medicine Field and traditional medicines of the Middle East}

\author{
Eric Manheimer, $\mathrm{PhD}$ \\ Research Associate, University of Maryland School of Medicine \\ Coordinator and Methodologist, Cochrane Collaboration Complementary Medicine Field \\ University of Maryland School of Medicine
}

Correspondence to: emanheimer@som.umaryland.edu

The Cochrane Collaboration is an international organization whose mission is to prepare, maintain, and promote the accessibility of systematic reviews of the benefits and risks of health care interventions. The Cochrane Complementary Medicine Field ("CAM Field") is the member group within the Collaboration focused on facilitating Cochrane reviews of complementary and alternative medicine (CAM). An important subset of CAM is traditional medicine, defined by the World Health Organization as "the sum total of knowledge, skills and practices based on the theories, beliefs and experiences indigenous to different cultures that are used to maintain health, as well as to prevent, diagnose, improve or treat physical and mental illnesses." Different countries often have their own forms of traditional medicine (e.g., traditional Chinese medicine, traditional Korean medicine). Many traditional medicine interventions have anecdotal support and preliminary evidence of effect. However, before traditional medicine interventions (as well as conventional medicine interventions) can be integrated into existing healthcare services, evidence of their effectiveness must first be documented through well-conducted systematic reviews of randomized controlled trials (RCTs), especially in this era of evidence-based medicine.

Identification of the relevant RCTs is an initial step in preparing systematic reviews. However, RCTs of traditional medicine are often inaccessible to Western researchers. This is because traditional medicine RCTs are often published in difficult to access regional or national databases and journals, and in languages other than English. The Cochrane CAM Field has therefore established collaborations with colleagues in China, Japan, Korea, India, and South Africa to identify traditional medicine RCTs published in these regions. To date, more than 10,000 citations of traditional medicine RCTs have been identified and registered on the Cochrane CAM trials database as a result of these collaborations. One unanswered question is whether there are any untapped RCT sources (e.g., databases, journals) focused on traditional Arabic medicines and published in the Middle East. If such sources exist, systematically searching through these sources to identify all relevant RCTs could facilitate the preparation of Cochrane reviews of traditional Arabic medicines.

While identifying RCTs is an important prerequisite for preparing Cochrane reviews, the main objective and work of the Cochrane Collaboration involves the actual preparation of Cochrane reviews. There are currently 656 CAM-related Cochrane reviews published in The Cochrane Library, which represents approximately $12 \%$ of the total number of Cochrane reviews (i.e., 5,570). Of the 656 CAM-related Cochrane reviews, 76 are reviews of traditional Chinese herbal medicine. However, there are no CAM-related Cochrane reviews of interventions specific to traditional Arabic medicine. Are there RCTs of any traditional Arabic medicine interventions? This is important to know because in the absence of high-quality RCTs, Cochrane reviews cannot draw any conclusions about a traditional medicine's effects.

One traditional Arabic medicine that has been evaluated with RCTs is black seed oil, which has been used for thousands of years in Middle Eastern countries for treating a variety of health ailments. Black seed oil has most commonly been used for treating asthma and allergies; however, it has also been used for treating digestive conditions, Type 2 diabetes, epilepsy and high blood pressure. A search of the Cochrane Central Register of Controlled Trials (CENTRAL) database retrieves 33 citations of RCTs on black seed oil. It therefore may be worth exploring whether there is any health condition (e.g., asthma) for which there is a sufficient number of black seed oil RCTs to warrant preparing a Cochrane review (e.g., "Black seed oil for asthma").

Additionally, one of the critiques of evidence-based medicine is that the drug industry funds and produces a large proportion of the RCTs that make up systematic reviews. As a result, clinical practice guidelines based on systematic reviews often focus on drugs and medical devices. This has been referred to as a "commissioning bias". Identifying traditional medicine RCTs, and preparing Cochrane reviews of these traditional medicine interventions, may help address this critique and increase the scope of coverage of Cochrane reviews. 